\title{
ACCURACY OF SATELLITE OPTICAL OBSERVATIONS AND PRECISE ORBIT DETERMINATION
}

\author{
L.Shakun, N.Koshkin, E.Korobeynikova, S.Strakhova, V.Dragomiretsky, \\ A.Ryabov, S.Melikyants, T.Golubovskaya, S.Terpan \\ Astronomical Observatory of Odessa I.I.Mechnikov National University, \\ Odessa, Ukraine, leomspace@gmail.com
}

\begin{abstract}
The monitoring of low-orbit space objects (LEO-objects) is performed in the Astronomical Observatory of Odessa I.I. Mechnikov National University (Ukraine) for many years. Decades-long archives of these observations are accessible within Ukrainian network of optical observers ( $\underline{\mathrm{UMOS}})$. In this work, we give an example of orbit determination for the satellite with the $1500-\mathrm{km}$ height of orbit based on angular observations in our observatory (Int. No. 086). For estimation of the measurement accuracy and accuracy of determination and propagation of satellite position, we analyze the observations of Ajisai satellite with the well-determined orbit. This allows making justified conclusions not only about random errors of separate measurements, but also to analyze the presence of systematic errors, including external ones to the measurement process. We have shown that the accuracy of one measurement has the standard deviation about 1 arcsec across the track and 1.4 arcsec along the track and systematical shifts in measurements of one track do not exceed 0.45 arcsec. Ajisai position in the interval of the orbit fitting is predicted with accuracy better than $30 \mathrm{~m}$ along the orbit and better than $10 \mathrm{~m}$ across the orbit for any its point.
\end{abstract}

Keywords: artificial satellite, LEO, optical observation, short-exposition observations, analysis of measuring precision, orbit estimation, Ajisai, Orekit, UMOS.

\section{Introduction}

The increase of occupation of the near-Earth orbits with the space debris constitutes a significant danger for active near-Earth satellites. The monitoring of near-Earth cosmic objects allows predicting the dangerous rendezvous of these objects. The optical observations of the objects on low-Earth orbits (LEO) are regularly performed in the Astronomical Observatory of Odessa I.I. Mechnikov National University (Ukraine) (Shulga et al., 2015).

For the observations, we use the telescope with the aperture of $50 \mathrm{~cm}$ and focal length $2 \mathrm{~m}$ on the azimuthal mounting. There is the television camera WACOM902H that works with the frequency of 50 interlace half-frame per second in the focal plane. Field of view of the telescope is about 10 arcmin. The LEO-objects are observed in tracking mode and this allows to obtain both the coordinate and the unique photometric information with high time resolution simultaneously (Koshkin et al., 2017). Shakun \& Koshkin (2014) give the detailed description of the methods of objects position measurements on the frame and time tagging of the frames. In that paper is shown that the accuracy of one measurement is about 0.8 arcsec and accuracy of time measurement from frame to frame is better than 0.0001 seconds.

However, in addition to the measurement accuracy caused by the method of frame measurements and features of the time tagging, observations may contain other errors that can be detected only by means of independent comparison of measurements and positions of the well-known object.

In this paper, we compare the accuracy of our measurements of Ajisai (EGS) position that has the welldetermined orbit with theoretical estimates of its visible position. We also estimate the orbit calculated by using our measurements with applying of the numerical model of satellite motion and compare the obtained orbit with the daily predictions that provided by International Laser Ranging Service (ILRS).

\section{The estimate of measurement accuracy}

To compare with our measurements, we need the estimates of satellite position that are significantly more precise than ones we get. The source of such estimates may be the prediction of Ajisai position provided by ILRS. For Ajisai such predictions are provided by Japan Aerospace Exploration Agency (JAXA) and NERC Space Geodesy Facility (see: CPF predictions). Every provider gives its prediction once a day for five days ahead. We joined successive predictions in one sequence and used only data of prediction for the first day (as the most reliable ones) for the calculation of the satellite position. So we gained these positions in the continuous range for the first eleven months of 2017. The comparison of the position in JAXA's range of prediction (jax) and ones of NERC Space Geodesy Facility (sgf) (Table 1) show that with rare exceptions difference between these predictions is less than $1 \mathrm{~m}$. In visible coordinates, it means that deviation between the predictions is less than 1 arcsec. We expect such predictions are suitable for our further analysis. We chose the JAX prediction, because it had smaller shifts of positions between daily forecasts and these shifts are also much less than $1 \mathrm{~m}$. 
Table 1: Comparison of Ajisai positions by JAX and SGP propagations for months of 2017. In the columns marked as ,std" the estimates of the standard deviation of the difference between predictions are given, in the columns, marked as "max abs" are maximal absolute values of differences. $\mathrm{dV}$ corresponds to the difference along the velocity vector, $\mathrm{dR}$ - in the perpendicular direction to the velocity vector in the orbital plane, $\mathrm{dN}$ - in the perpendicular direction to the orbital plane.

\begin{tabular}{|c|c|c|c|c|c|c|}
\hline \multirow{2}{*}{$\begin{array}{c}\text { Year. } \\
\text { Month }\end{array}$} & \multicolumn{3}{|c|}{ std } & \multicolumn{3}{|c|}{ max abs } \\
\cline { 2 - 7 } & $\begin{array}{c}\mathbf{d V} \\
(\mathbf{m})\end{array}$ & $\begin{array}{c}\mathbf{d R} \\
(\mathbf{m})\end{array}$ & $\begin{array}{c}\mathbf{d N} \\
(\mathbf{m})\end{array}$ & $\begin{array}{c}\mathbf{d V} \\
(\mathbf{m})\end{array}$ & $\begin{array}{c}\mathbf{d R} \\
(\mathbf{m})\end{array}$ & $\begin{array}{c}\mathbf{d N} \\
(\mathbf{m})\end{array}$ \\
\hline 2017.01 & 0,305 & 0,080 & 0,311 & 1,532 & 0,248 & 0,909 \\
\hline 2017.02 & 0,254 & 0,045 & 0,324 & 1,151 & 0,138 & 0,958 \\
\hline 2017.03 & 0,245 & 0,049 & 0,299 & 1,520 & 0,184 & 0,796 \\
\hline 2017.04 & 1,408 & 0,072 & 0,260 & 9,006 & 0,348 & 0,679 \\
\hline 2017.05 & 0,256 & 0,043 & 0,284 & 1,154 & 0,164 & 0,747 \\
\hline 2017.06 & 0,449 & 0,076 & 0,324 & 2,562 & 0,220 & 0,904 \\
\hline 2017.07 & 0,282 & 0,050 & 0,340 & 2,519 & 0,211 & 1,076 \\
\hline 2017.08 & 0,229 & 0,067 & 0,328 & 1,002 & 0,224 & 1,127 \\
\hline 2017.09 & 0,495 & 0,072 & 0,493 & 2,733 & 0,224 & 1,557 \\
\hline 2017.10 & 0,241 & 0,038 & 0,251 & 0,863 & 0,144 & 0,901 \\
\hline 2017.11 & 0,600 & 0,060 & 0,259 & 2,691 & 0,233 & 0,879 \\
\hline
\end{tabular}

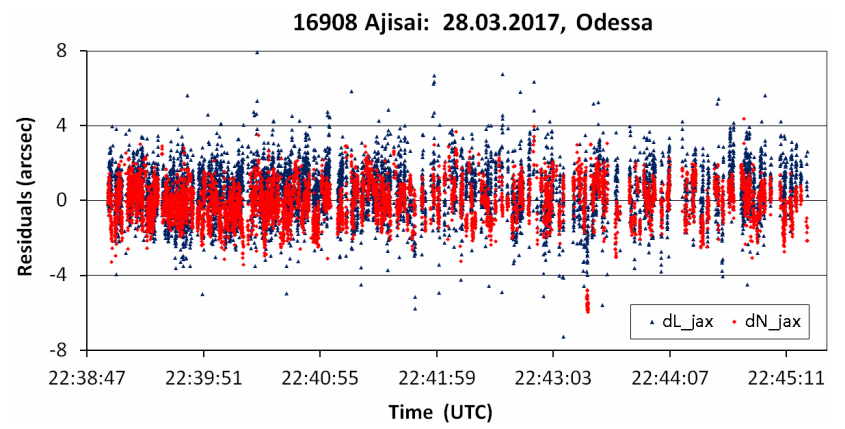

Figure 1: Residuals between measurements and calculated visible coordinates of Ajisai according to the prediction of JAX for 28.03.2017. dL are the residuals along the visible trajectory, $\mathrm{dN}$ are the residuals across the visible trajectory.

In Figure 1 the residuals between measurements and calculated visible coordinates according to the prediction of JAX are shown for the typical satellite track. The residuals are considered in terms of along the visible trajectory and across it. The coordinate system necessary for decomposition of the vector of residuals can be obtained on the basis of theoretical topocentric values of position vector (r) and velocity vector (v) of a satellite as

$$
\vec{i}=\frac{\vec{r}}{r}, \vec{j}=[\vec{k}, \vec{i}], \quad \vec{k}=\frac{[\vec{r}, \vec{v}]}{|[\vec{r}, \vec{v}]|},
$$

where unit vector $\boldsymbol{i}$ is directed along the vector observersatellite, unit vector $\boldsymbol{j}$ is codirectional to the tangent line to the visible trajectory and unit vector $\boldsymbol{k}$ is perpendicular to visible trajectory in given point. The visible angular velocity of a satellite is determined by projecting of velocity vector on the vector $\boldsymbol{j}$.

This coordinate system for representation of the residuals usually is better connected with the causes of errors appearance and it allows to understand them easier than the traditional representation of residuals in the equatorial or the horizontal system of coordinates.

Since when we observe LEO-objects their visible angular velocity is high, the images of reference stars or the objects become drawn-out along the motion direction and this requires the correct alignment of the position of the center or the edges of the stroke with the moment of time. In addition, due to the high angular velocity of the object, even small errors in registration of moments of time when the image was taken, lead to significant shifts in the estimate of coordinates along the trajectory. Both causes lead to the perceptible difference in behavior of errors along and across the trajectory. Among other things, detecting of the systematic errors in time registration is the most difficult if we consider the observations solely.

For the estimation of measurement accuracy and estimation of the orbit, we take 13 tracks of Ajisai from 23.03.2017 to 12.04.2017. In Table 2 the statistical parameters of residuals between observations and theoretical values based on the JAX predictions are given separately for every track.

One can see, that the estimate of the standard deviation across the track is stable in the range from 0.9 to 1.1 arcsec, but the error along the track is 1.4 times higher. The mean of all residuals of the whole track usually is significantly biased from zero. The theoretical difference of the mean bias of residuals between two predictions (sgf-jax) is given in Table 1 for comparison. There the residuals are calculated for the same moment of time when the observations were made. Thus, it is obvious, that obtained biases of the tracks cannot be explained by differences in predictions assuming a prediction error is of the order of $1 \mathrm{~m}$. For the explanation of these mean biases, the residuals between satellite positions and theoretical predictions must be about $10 \mathrm{~m}$ and more.

In the end, we give the estimates of biases in terms of time moments shifts along the trajectory dT that accentuates the extreme importance of thorough measuring of the timing of images obtaining. Taking into account, that Ajisai is the satellite with relatively slow visible velocity, we expect that for very low orbit satellites these requirements will be even more essential.

\section{The estimation of orbit}

Now, for considering the observations presented in Table 2 , we estimate the satellite's orbit using the numerical model of motion.

For integration of equations of motion and astrometric transformations, we used low-level astrodynamics library Orekit 9.0. But for the model of observations and in the algorithm of optimization of the satellite motion model we used our own code in the programming language Kotlin. 
Table 2: Statistics of measurements of the Ajisai positions. dL are the residuals along the trajectory; dN are residuals across the trajectory; dT are residuals along the trajectory in terms of time-lags. avg are mean values of bias for the whole track; std are the estimate of the standard deviation for one measurement; sgf-jax are the mean values of residuals between two theoretical ILRS-predictions.

\begin{tabular}{|l|r|r|r|r|r|r|r|r|r|}
\hline \multirow{2}{*}{$\begin{array}{c}\text { Date and Time } \\
\text { of Track }\end{array}$} & \multirow{2}{*}{$\begin{array}{c}\text { Numbers of } \\
\text { measurements }\end{array}$} & \multicolumn{3}{|c|}{ dL (arcsec) } & \multicolumn{3}{c|}{ dN (arcsec) } & \multicolumn{2}{c|}{ dT (sec) } \\
\cline { 3 - 10 } & & avg & std & sgf-jax & avg & \multicolumn{1}{c|}{ std } & \multicolumn{1}{c|}{ sgf-jax } & \multicolumn{1}{c|}{ avg } & \multicolumn{1}{c|}{ sgf-jax } \\
\hline $23.03 .201720: 03$ & 7483 & 0,0019 & 1,32 & 0,0010 & $-0,2710$ & 0,95 & 0,0410 & 0,00001 & 0,000001 \\
\hline $28.03 .201720: 35$ & 10273 & 0,1294 & 1,25 & $-0,0101$ & $-0,4648$ & 1,00 & 0,0164 & 0,00010 & $-0,000017$ \\
\hline $28.03 .201722: 38$ & 7482 & 0,4377 & 1,32 & $-0,0069$ & $-0,1131$ & 1,00 & 0,0072 & 0,00059 & $-0,000009$ \\
\hline $29.03 .201721: 43$ & 6808 & 0,2556 & 1,29 & 0,0006 & $-0,1398$ & 0,99 & 0,0188 & 0,00032 & 0,000002 \\
\hline $30.03 .201720: 48$ & 7670 & 0,2906 & 1,21 & 0,0092 & $-0,2450$ & 0,90 & 0,0069 & 0,00040 & 0,000013 \\
\hline $31.03 .201720: 01$ & 2460 & 0,4214 & 1,30 & 0,0035 & $-0,7009$ & 1,09 & 0,0047 & 0,00073 & 0,000005 \\
\hline $31.03 .201721: 58$ & 8156 & 0,3957 & 1,35 & 0,0152 & 0,0876 & 1,05 & $-0,0053$ & 0,00056 & 0,000021 \\
\hline $03.04 .201721: 17$ & 10555 & 0,3479 & 1,40 & 0,0041 & $-0,0574$ & 1,08 & 0,0115 & 0,00048 & 0,000005 \\
\hline $10.04 .201721: 02$ & 5435 & 0,3074 & 1,28 & $-0,0008$ & 0,3626 & 0,88 & $-0,0203$ & 0,00043 & $-0,000001$ \\
\hline $10.04 .201723: 03$ & 7168 & $-0,0057$ & 1,15 & 0,0054 & 0,4526 & 1,05 & $-0,0267$ & $-0,00001$ & 0,000010 \\
\hline $11.04 .201720: 16$ & 1667 & 0,0091 & 1,52 & 0,0008 & $-0,2164$ & 1,09 & $-0,0240$ & $-0,00012$ & 0,000002 \\
\hline $11.04 .201722: 09$ & 10610 & 0,1735 & 1,31 & 0,0121 & 0,1367 & 1,11 & $-0,0233$ & 0,00027 & 0,000020 \\
\hline $12.04 .201721: 16$ & 7050 & 0,3338 & 1,28 & 0,0177 & 0,1667 & 0,95 & $-0,0185$ & 0,00050 & 0,000025 \\
\hline
\end{tabular}

The process of orbit determination includes the following stages:

1. A primary estimate of Kepler orbits parameters from three observations for one track.

2. Optimization of the Keplerian orbit for all observations of the single track.

3. Optimization of position and velocity for a given epoch for the numerical model of satellite motion for one track.

4. Optimization of the position and velocity of the satellite for a given epoch for the numerical model of satellite motion for one or several tracks on close days

5. Optimization of the position, the velocity of the satellite for a given epoch, and parameters of the ratio of cross-section to mass, in terms of correction for the influence of sunlight pressure and correction for the influence of the atmosphere.

Thus, to determine the orbit, we did not use any a priori information about the satellite's orbital motion.

In the numerical integration of the equations of satellite motion, the following perturbing forces were taken into account:

1. The Earth gravity field was calculated by using the model Eigen6s (Furste et al., 2011) truncated to the 51 st degree and the 51 st order. The contribution of the harmonics was derived in accordance with the algorithm Holmes \& Featherstone (2002).

2. Ocean tides in accordance with the model FES2004 (Lyard et al., 2006).

3. Gravitational perturbation from the Moon and the Sun according to the model DE-430.

4. Tides in the solid body of the Earth from the Moon and the Sun.

5. The force of solar radiation pressure with considering umbra and penumbra regions in the term of an effective cross-section of the satellite.
6. Atmospheric drag force in accordance with DTM2000 (Bruinsma et al., 2003) in the term of the effective cross-section. The level of the solar radiation flux was taken as an average one.

7. The general relativity term.

For integrating the equation, we used the method Dormand-Prince 5(3) (Hairer et al., 2011), with the variable step and accuracy control $0.001 \mathrm{~m}$ on a step and the maximal value of step $120 \mathrm{sec}$.

The effective cross-sections of the satellite fitted independently in cases of solar radiation pressure and the atmospheric drag force.

The results of these fitting are presented in Figure 2 in the form of differences between the satellite position $(\mathrm{dV}$, $\mathrm{dR}, \mathrm{dN}$ ) according to the orbit optimized by us and the positions predicted by JAX.

As one can see, in the interval of fitting, the differences along the satellite trajectory are not more than $30 \mathrm{~m}$, and in the perpendicular direction are not more than $10 \mathrm{~m}$.

In the month interval of the propagation, the differences along the trajectory are not more than $100 \mathrm{~m}$. And only a month later the differences have a linear trend along the orbit. Three months after the fitting interval, the time lag of the satellite along the orbit is about $0.4 \mathrm{sec}$.

\section{Discussion and conclusion}

Thus we have shown that

- The accuracy of the one measurement of object positions across the visible trajectory is from 0.9 to 1.1 arcsec, and along the visible path from 1.2 to 1.4 arcsec.

- The mean values of the residuals of the coordinates both along and across the visible trajectory are of several tenths of an arcsecond and require the further analysis and elimination. 


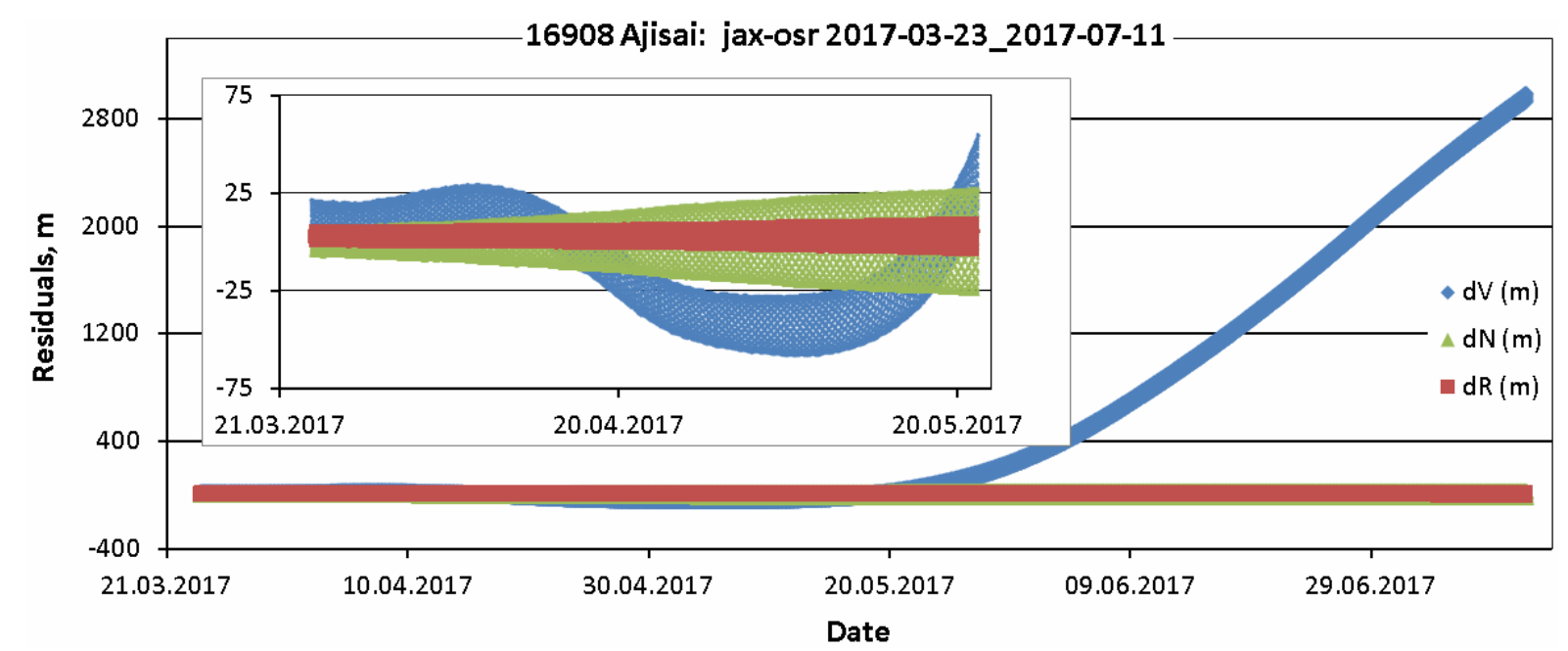

Figure 2: The difference between the positions of the satellite according to the optimized orbit and positions according to the prediction of JAX. $\mathrm{dV}$ are the differences along the direction of the velocity vector; $\mathrm{dN}$ are the differences in the perpendicular direction to the instantaneous plane of the orbit; $\mathrm{dR}$ are the differences in the perpendicular direction to the velocity vector in the instantaneous plane of the orbit.

- Observations of such quality make possible to predict positions within the interval of fitting with accuracy to dozens of meters along the orbit and better than 10 meters in the perpendicular directions to the orbit.

- The monthly prediction of the satellite positions is suitable for planning automated observations.

The causes of the significant bias of the mean residuals from zero can be both systematic biases along the orbit and the correlation of close observations. Taking to account the similarity of the value of bias along and across the orbit in our case, most likely we deal with the second cause. Sure, very short exposures and a small field of view of the telescope results in a small number of reference stars, that in many frames does not allow to restore the coordinate system completely. The use of the mean coordinate system moving from frame to frame without the doubt should result in a correlation of close measurements. The result of this is the significant bias of the mean values of the residuals from zero.

The conclusions of Section 2 on the accuracy of the measurements are in good agreement with the residuals between the fitting orbit and the JAX prediction obtained in Section 3. Relatively larger deviations in the difference of positions along the orbits are probably related to the fact that all observations were obtained at one observation point in approximately the same time of day, and this fact allows to see only a small part of the orbit.

After 2-3 months, the difference between the predicted and the real position of the satellite along the orbit grows up to several arcminutes, which is quite high and can go beyond the field of view of the telescope. However, almost whole deviation lies along the visible motion of the satellite. Correction of the initial moment of observations allows us to remove the major part of the accumulated deviation along the orbit. Thus, the prediction based on our observa- tions can be successfully used to detect and to track the satellite for several months later than the orbit was fitted.

Acknowledgements. We are thankful to the authors of Orekit for the development and long-term development improvement of such useful engineering solution. Without Orekit, this work would take considerably greater effort.

\section{References}

Bruinsma S., Thuillier G., Barlier F.: 2003, Journal of Atmospheric and Solar-Terrestrial Physics, 65, 1053.

CPF predictions:

ftp://edc.dgfi.tum.de/pub/slr/cpf_predicts/2017/ajisai/.

EGS (Ajisai): http://global.jaxa.jp/projects/sat/egs/.

Hairer E., Norsett S., Wanner G.: 1993, Solving Ordinary Differential Equations (Springer), 528.

Holmes S., Featherstone W.: 2002, Journal of Geodesy, 76, 279.

ILRS: https://ilrs.cddis.eosdis.nasa.gov/.

Furste C., Bruinsma S., Shako R. et al.: 2011, Geophysical Research Abstracts, 13, EGU2011-3242-2.

Kaminski K., Koshkin N., Shakun L. et al.: 2017, Proc. of 7th Eur. Conf. on Space Debris, ESA/ESOC, Darmstadt, 18-21 Apr. 2017. Available at: (https://conference.sdo.esoc.esa.int/proceedings/sdc7/ paper/721/SDC7-paper721.pdf).

Koshkin N. et al.: 2017, Adv. in Space Res., 60, 1389.

Lyard F. et al.: 2006, Ocean Dynamics, 56, 394.

Orekit: https://www.orekit.org/.

Shakun L., Koshkin N.: 2014, Adv. in Space Res., 53, 1834. Shulga A.V. et al.: 2015, Space Science and Technology, 21, 3, 74 .

UMOS: http://umos.mao.kiev.ua/eng/, https://en.wikipedia.org/wiki/Ukrainian_Optical_Facilit ies_for_Near-Earth_Space_Surveillance_Network. 